\title{
A Rare Case of an Ectopic Pregnancy in the Pouch of Douglas and a Review of Abdominal Ectopic Pregnancies
}

\author{
Thomas Owain Gabbott ${ }^{1}$, Parveen Verasingam ${ }^{2}$ \\ Department of Obstetrics and Gynaecology, ${ }^{1}$ Leeds University, United Kingdom; ${ }^{2}$ Airedale General Hospital, Keighley, BD20 6TD, \\ West Yorkshire, United Kingdom.
}

\section{Corresponding Author: \\ Dr. Parveen Verasingam \\ Email: umpkv@leeds.ac.uk}

This is an Open Access article distributed under the terms of the Creative Commons Attribution License (creativecommons.org) licenses/by/3.0).

Received : January 6, 2019

Accepted : June 3, 2019

Published : June 20, 2019

\begin{abstract}
Introduction: It is predicted that the prevalence of abdominal ectopic pregnancy ranges between 1:10,000 to $1: 30,000$ pregnancies. Maternally mortality is high, at an estimated $5-18 \%$. In this report, we detail a case of an ectopic pregnancy implanted in the pouch of Douglas secondary to tubal abortion. Case Report: A 29 year old pregnant female presented to Accident \& Emergency with a 12 hour history of severe, constant right iliac fossa pain and vaginal bleeding. Patient had severe tenderness in the right adnexa. Her beta human chorionic gonadotropin serum level was 31,382 IU/L. Ultrasound scan revealed a mass next to the uterus. A provisional diagnosis of an ectopic pregnancy was made. Ovarian malignancy and gestational trophoblastic disease were also considered. Diagnostic laparoscopy revealed large complex mass in the pouch of Douglas which was removed. She recovered well following the surgery. Conclusion: Ultrasound remains the gold standard for investigating an ectopic pregnancy or to rule out other differentials that can clinically present in the same manner. However, as only $50 \%$ accuracy can be assumed when diagnosing an early abdominal ectopic with ultrasound, a high index of suspicion is required. A diagnostic laparoscopy is invaluable in diagnosis and management of an ectopic pregnancy.
\end{abstract}

Keywords: Infant, Melanoma, Neurocutaneous Melanosis, Nevus, Pregnancy, Skin Neoplasms.

\section{Introduction}

It is predicted that the prevalence of abdominal ectopic pregnancy ranges between 1:10,000 to 1:30,000 pregnancies [1]. Maternally mortality is high, at an estimated 5-18\% [1]. In this report, we detail a case of an ectopic pregnancy implanted in the pouch of Douglas (POD) secondary to tubal abortion.

\section{Case Report}

A 29 year old female presented to Accident \& Emergency with a 12 hour history of severe, constant right iliac fossa pain and vaginal bleeding. The pain was associated with nausea and vomiting. There was no shoulder tip pain. Urinary pregnancy test was positive. She is gravida 5 and parity 4 and she had a normal vaginal delivery 14 weeks before she presented to the department. Her last menstrual period was thought to be around six weeks ago. For the last three weeks however, she described light on and off brown discharge. Fresh vaginal bleeding was reported 4 hours prior to admittance. Apart from a large loop excision of the transformation zone (LLETZ) procedure two years ago for an abnormal smear result, she had no other past medical history. She was not using any contraception.

An abdominal examination revealed guarding and rebound tenderness in the right iliac fossa. All her observations were within normal 
limits including temperature. Vaginal examination discovered no adnexal masses however the patient had tenderness in the right adnexa. There was no cervical excitation. A speculum inspection noted a healthy cervix and small amount of bleeding. Her routine blood tests were all within normal range. She was rhesus negative with a negative antibody screen. Her beta human chorionic gonadotropin (ß-HCG) serum level was 31,382 IU/L. Bedside transabdominal ultrasound scan revealed moderate amount of free fluid in the pelvis and no intrauterine pregnancy. There was a $6 \mathrm{~cm}$ mass adjacent to the uterus. She was admitted into the ward and a departmental ultrasound was requested to rule out other differential diagnosis which included ovarian malignancy and gestational trophoblastic disease (GTD).

The following morning, she had a departmental ultrasound which revealed a complex mass to the right of the uterus measuring $60 \mathrm{~mm}$ in addition to a second echogenic area measuring $32 \mathrm{~mm}$ adjacent to the mass which may represent a gestational sac [Fig.1]. There was also a moderate to large amount of free fluid within the pelvis. A diagnosis of an ectopic pregnancy was made. We proceeded to perform diagnostic laparoscopy following the scan. Upon entry into the abdominal cavity, there was a litre of blood which was removed. A large complex mass was seen in the pouch of Douglas (POD) and was removed via the suction device [Fig.2]. The right tube appears to be swollen

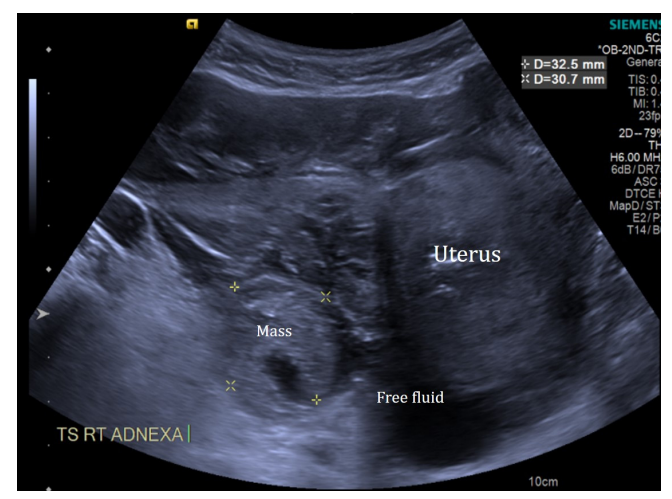

Fig.1: Transvaginal ultrasound revealing a complex mass next to the uterus (right side). There was a moderate amount of free fluid within the pelvis. and we proceeded to perform salpingectomy. The right uterosacral ligament appeared to be bisected by the invading mass [Fig.3]. We concluded that she had a tubal abortion and the ectopic pregnancy kept on growing in the POD. We continued to perform peritoneal lavage and manage to gently peel off decidua-like structures from the POD. She recovered well following the surgery was discharged home the next day. She returned to the gynaecological day unit for further $\beta-H C G$ testing [Table 1].

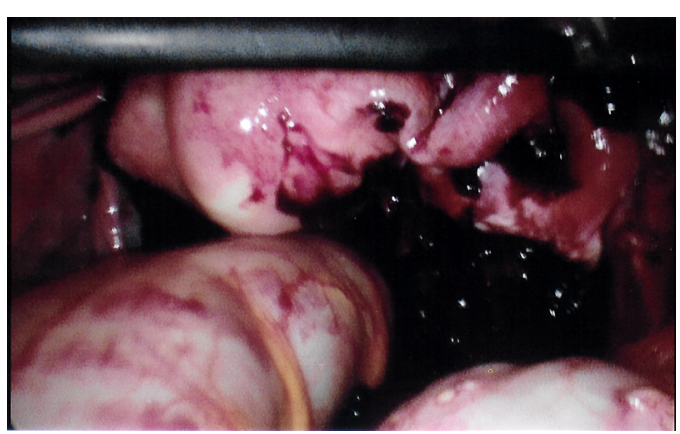

Fig.2: Complex mass seen in the pouch of Douglass with organized blood clot.

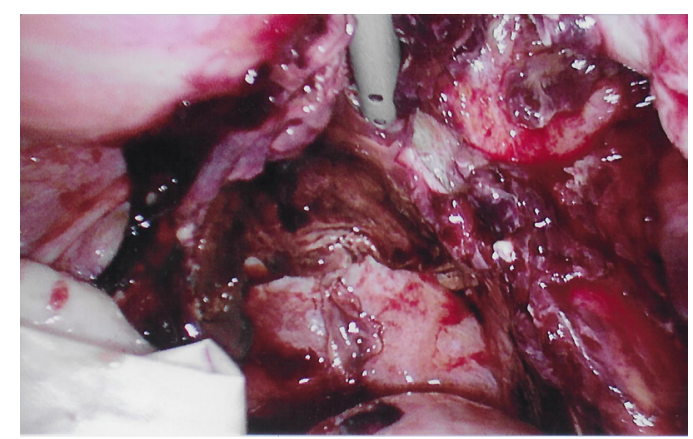

Fig.3: Site of implantation seen on the right utero-sacral ligament. The complex ectopic mass has been removed.

Table 1: $\beta$ HCG results before and after surgery.

\begin{tabular}{|l|l|}
\hline \multicolumn{1}{|c|}{ Day } & \multicolumn{1}{c|}{ B-HCG (IU/L) } \\
\hline Pre-operative & 31,382 \\
\hline Day 1 post-operative & 8,535 \\
\hline Day 7 post-operative & 369 \\
\hline Day 14 post-operative & 52 \\
\hline Day 21 post-operative & 13 \\
\hline Day 28 post-operative & $<5$ \\
\hline
\end{tabular}




\section{Discussion}

Ectopic pregnancy occurs in roughly $1-2 \%$ of all pregnancies with $95 \%$ of them occurring in the fallopian tube [1]. Only $1 \%$ of all ectopics are thought to manifest in the abdomen. Consequently, it is predicted that 1:10,000 - 1:30,000 pregnancies result in abdominal implantation [2,3]. If this is the case, maternally mortality is high, at an estimated $5-18 \%$ [3]. Thus, quick and accurate diagnosis of an abdominal pregnancy is crucial.

Secondary implantation in the POD following a tubal abortion is rare. An extensive literature review of Embase (1996 to 2018) and Medline (1946 to 2018) revealed only two documented cases that are similar to this clinical scenario [4]. When the search was widened to include ruptured tubal ectopics with secondary implantation in the POD, a further two cases were reported $[5,6]$. Yet, contrary to the available literature, a 45-year review demonstrated that the POD is the most common sight for an abdominal pregnancy to attach $(24.3 \%$ of all abdominal ectopic pregnancies) [7]. Other documented locations of abdominal pregnancies include attachment to the liver, spleen and appendix [8-10]. These are reported more infrequently than POD implantation.

A differential diagnosis of ovarian cancer is vital to rule out when exploring the possibility of an ectopic pregnancy. Both have the possibility to present as lower abdominal pain and vaginal bleeding with a solid mass next to the uterus and free fluid surrounding the adnexa on ultrasound.

It is believed that whilst adnexal masses occur in roughly 1:800 pregnancies, the prevalence of ovarian cancers in pregnancy has been reported as 1:653 [11,12]. Thus, although rare, ovarian tumors must be considered as a cause of the symptoms mimicking an ectopic pregnancy presentation. Cases of ruptured ovarian tumors being misdiagnosed as ruptured ectopic pregnancies until laparoscopy and pathological analysis was performed have been reported [13].
Furthermore, it is crucial to suspect gestational trophoblastic disease (GTD). This is a term used for a collection of tumors that can grow in the uterus. Presentation of both ectopic pregnancies and GTD can be remarkably similar; lower abdominal pain accompanied with vaginal bleeding - particularly if hemoperitoneum is present as well. However, as a consequence of the gestational nature of the disease, it is possible for patient's to develop GTD following an ectopic pregnancy. Studies have reported mixed prevalence of this, ranging from $0.16: 1,000$ deliveries to $1.56: 1,000[14,15]$. This equates to roughly an $18 \%$ chance of GTD in ectopic pregnancy.

Ultrasound remains the gold standard for investigating an ectopic pregnancy. However, as only $50 \%$ accuracy can be assumed when diagnosing an early abdominal ectopic with ultrasound, a high index of suspicion is required [16]. When the location of the pregnancy is still uncertain, magnetic resonance imaging should be utilized. As with all ectopic pregnancies (regardless of location), they can be managed either medically or surgically (or both). Agents such as methotrexate and potassium chloride have been demonstrated to successfully treat an early abdominal pregnancy $[17,18]$. As with this case diagnostic laparoscopy is invaluable in diagnosis and management of an ectopic of unknown location.

Surgically treating known abdominal pregnancies, or any ectopic pregnancy that has a large gestational age, should be managed by a senior multi-disciplinary team. This is because of the potential risk of life-threatening bleeding during the operation.

\section{Conclusion}

Whilst secondary implantation in the POD following a tubal abortion is the most common sight for implantation for abdominal ectopic pregnancies, their prevalence remains extremely rare and should be managed with vigilance, expertise and in a 
timely manner. Maternal mortality is high when ectopic pregnancies attach in the abdomen and senior healthcare staff should always have a high index of suspicion when diagnosing an ectopic of unknown location - both in terms of investigating the patient's signs and symptoms and ruling out other differential diagnoses.

Contributors: TOG: manuscript writing, patient management; PV: manuscript editing, patient management. PV will act as guarantor. Both authors approved the final version of this manuscript.

Funding: None; Competing interests: None stated.

\section{References}

1. Jain V, Jain S, Jain S, Rughuwanshi J. A rare case of secondary abdominal pregnancy that resulted in a healthy newborn. Journal of Obstetrics \& Gynaecology of India. 2014;64(6):430-432.

2. Atrash HK, Friede A, Hogue CJ. Abdominal pregnancy in the United States: frequency and maternal mortatilty. Obstetrics and Gynaecology. 1987;63:333-337.

3. Worley KC, Hnat MD, Cunningham FG. Advanced extra uterine pregnancy: diagnostic and therapeutic challenges. American Journal of Obstetrics and Gynaecology. 2008;198(3):e1-7.

4. Abdul-Jabbar NAR, Saquib S, Talha W.E.M. Successful management of abdominal pregnancy: Two Case Reports. Oman Medical Journal. 2018;33(2):171-175.

5. Giannopoulous T, Katesmark M. Ruptured tubal ectopic pregnancy with secondary implantation in the pouch of Douglas. Journal of Obstetrics and Gynaecology. 2004;2(2):199-200.

6. Rohde E, Frahm R. A case of secondary abdominal pregnancy. Zentralblatt fur Gynakologie. 1989;111(8):530-532.

7. Poole A., Hass D, Mangann EF. Early abdominal ectopic pregnancies: a systematic review of the literature. Gynaecology and Obstetrics Investigation. 2012;74(4):249-260.

8. Yagil Y, Beck-Razi N, Amit A, Kerner H, Gaitini D.
Splenic pregancy: the role of abdominal imaging. J Ultrasound Med. 2007;26(11):1629-1632.

9. Rosso M, Sijanovic S, Topolovec Z, Vidosavljevic D, Selthofer R, Blazicevic V. Secondary abdominal appendicular pregnancy: Case Report. Srpski Arhiv Za Celokupno Lekarstvo. 2014;142(7-8):484-487.

10. Chui AKK., Lo KWK, Choi PCL, Sung MCC. Primary hepatic pregnancy. ANZ Journal of Surgery. 2001;71(4):260-261.

11. Pennes DR, Bowerman RA. Silver TM. Echogenic adnexal masses assocaited with first-trimester pregnancy: sonographic appearance and clinical significance. Journal of Clinical Ultrasound. 1985;13(6):391-396.

12. El-Yahia AR, Rahman J, Rahman MS, al-Suleiman SA. Ovarian tumours in pregnancy. The Australian and New Zealand Journal of Obstetrics and Gynaecology. 1991;31(4):327-330.

13. Riley GM, Babcook C, Jain K. Ruptured malignant ovarian tumor mimicking ruptured ectopic pregnancy. J Ultrasound Med. 1996;15(12):871-873.

14. Tasha I, Kroi E, Karameta A, Shahinaj R, Manoku N. Prevalence of gestational trophoblastic disease in ectopic pregnancy. Journal of Prenatal Medicine. 2010;4(2): 26-29.

15. Cortes-Charry R, Figueira, LM, Garcia-Barriola V, Gomez C, Garcia I, Santiago C. Gestional trophoblastic disease in ectopic pregnancy: A case series. The Journal of Reproductive of Reproductive Medicine. 2006;51(10):760-763.

16. Agarwal N, Odejinmi, F. Early abdominal ectopic pregnancy: challenges, update and review of current management. The Obstetrician \& Gynaecologist. 2014;16(3):193-198.

17. Anderson PM, Opfer EK, Busch JM. Magann EF. An early abdominal wall ectopic pregnancy successfully treated with ultrasound guided intralesional methotrexate: A case report. Obstetrics and Gynaecology International. 2009; Article ID:247452.

18. Schieber MD, Cedars MI. Case report: Successful non-surgical management of a heterotopic abdominal pregnancy following embryo transfer with cryopreserved-thawed embryos. Human Reproductive. 1999;14(5):1375-1377. 